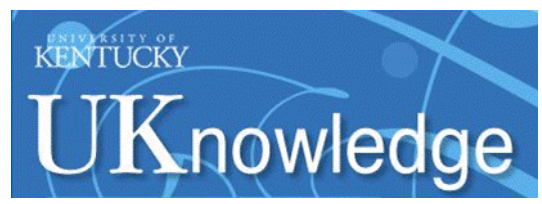

University of Kentucky

UKnowledge

\title{
Rationale and Methods for a Multicenter Clinical Trial Assessing Exercise and Intensive Vascular Risk Reduction in Preventing Dementia (rrAD Study)
}

\author{
Amanda N. Szabo-Reed \\ University of Kansas \\ Eric Vidoni \\ University of Kansas \\ Ellen F. Binder \\ Washington University in St. Louis \\ Jeffrey Burns \\ University of Kansas \\ C. Munro Cullum

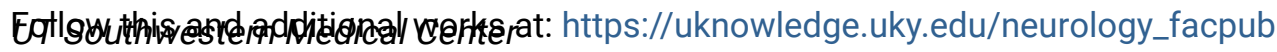 \\ Part of the Internal Medicine Commons, Neurology Commons, and the Psychiatry and Psychology \\ seemmext page for additional authors \\ Right click to open a feedback form in a new tab to let us know how this document benefits you.
}

\section{Repository Citation}

Szabo-Reed, Amanda N.; Vidoni, Eric; Binder, Ellen F.; Burns, Jeffrey; Cullum, C. Munro; Gahan, William P.; Gupta, Aditi; Hynan, Linda S.; Kerwin, Diana R.; Rossetti, Heidi; Stowe, Ann M.; Vongpatanasin, Wanpen; Zhu, David C.; Zhang, Rong; and Keller, Jeffrey N., "Rationale and Methods for a Multicenter Clinical Trial Assessing Exercise and Intensive Vascular Risk Reduction in Preventing Dementia (rrAD Study)" (2019). Neurology Faculty Publications. 61.

https://uknowledge.uky.edu/neurology_facpub/61

This Article is brought to you for free and open access by the Neurology at UKnowledge. It has been accepted for inclusion in Neurology Faculty Publications by an authorized administrator of UKnowledge. For more information, please contact UKnowledge@lsv.uky.edu. 


\title{
Rationale and Methods for a Multicenter Clinical Trial Assessing Exercise and Intensive Vascular Risk Reduction in Preventing Dementia (rrAD Study)
}

\author{
Digital Object Identifier (DOI) \\ https://doi.org/10.1016/j.cct.2019.02.007 \\ Notes/Citation Information \\ Published in Contemporary Clinical Trials, v. 79. \\ Copyright @ 2019 . Published by Elsevier Inc. \\ (c) 2019. This manuscript version is made available under the CC-BY-NC-ND 4.0 license \\ https://creativecommons.org/licenses/by-nc-nd/4.0/
}

\section{Authors}

Amanda N. Szabo-Reed, Eric Vidoni, Ellen F. Binder, Jeffrey Burns, C. Munro Cullum, William P. Gahan, Aditi Gupta, Linda S. Hynan, Diana R. Kerwin, Heidi Rossetti, Ann M. Stowe, Wanpen Vongpatanasin, David C. Zhu, Rong Zhang, and Jeffrey N. Keller 
Published in final edited form as:

Contemp Clin Trials. 2019 April ; 79: 44-54. doi:10.1016/j.cct.2019.02.007.

\title{
Rationale and Methods for a Multicenter Clinical Trial assessing Exercise and Intensive Vascular Risk Reduction in Preventing Dementia (rrAD Study)
}

\author{
Amanda N. Szabo-Reed ${ }^{a, b}$, Eric Vidoni ${ }^{a, c}$, Ellen F. Binder ${ }^{d}$, Jeffrey Burns ${ }^{a, c}$, C. Munro \\ Cullum $^{e, f}$, William P. Gahan' ${ }^{g}$, Aditi Gupta ${ }^{a, b}$, Linda S. Hynan ${ }^{e, h}$, Diana R. Kerwin ${ }^{i, j}$, Heidi \\ Rossetti $^{\mathrm{e}}$, Ann M. Stowe ${ }^{\mathrm{k}}$, Wanpen Vongpatanasing, David C. Zhu', Rong Zhang ${ }^{\mathrm{i}, \mathrm{f}}$, and \\ Jeffrey N. Kellerg \\ a.KU Alzheimer's Disease Center, Fairway, KS, USA. \\ b. Department of Internal Medicine, University of Kansas Medical Center, Kansas City, KS, USA \\ c.Department of Neurology, University of Kansas Medical Center, Kansas City, KS, USA \\ d.Department of Internal Medicine, Division of Geriatrics \& Nutritional Science, Washington \\ University School of Medicine in St. Louis, St. Louis, MO, USA \\ e.Department of Psychiatry, UT Southwestern Medical Center, Dallas, TX, USA \\ f.Department of Neurology \& Neurotherapeutics, UT Southwestern Medical Center, Dallas, TX, \\ USA \\ 9.Institute for Dementia Research and Prevention, Pennington Biomedical Research Center, \\ Baton Rouge, LA, USA \\ h.Department of Clinical Sciences, UT Southwestern Medical Center, Dallas, TX, USA \\ i. Institute for Exercise and Environmental Medicine, Texas Health Presbyterian Hospital Dallas, \\ Dallas, USA \\ j.Kerwin Research Center and Memory Care, Dallas, TX, USA \\ k.Department of Neurology, University of Kentucky, Lexington, KY, USA \\ I.Department for Radiology, Michigan State University, East Lansing, MI, USA
}

\begin{abstract}
Alzheimer's Disease (AD) is an age-related disease with modifiable risk factors such as hypertension, hypercholesterolemia, obesity, and physical inactivity influencing the onset and progression. There is however, no direct evidence that reducing these risk factors prevents or slows
\end{abstract}

\footnotetext{
*Corresponding author: Jeffrey Keller, PhD, jeffrey.keller@pbrc.edu.

Co-author emails: Amanda N. Szabo Reed (aszabo@kumc.edu), Eric Vidoni (evidoni@kumc.edu), Ellen Binder

(ebinder@wustl.edu), Jeffrey Burns (jburns2@kumc.edu), Munro Cullum (munro.cullum@utsouthwestern.edu), William P. Gahan (william.gahan@pbrc.edu), Aditi Gupta (agupta@kumc.edu), Linda Hynan (Linda.Hynan@utsouthwestern.edu), Diana R. Kerwin (DianaKerwin@texashealth.org), Heidi Rossetti (heidi.rossetti@utsouthwestern.edu), Ann Stowe (Ann.Stowe@uky.edu), Wanpen Vongpatanasin (wanpen.vongpatanasin@utsouthwestern.edu), David C.Zhu (zhuda@msu.edu), Rong Zhang

(RongZhang@texashealth.org), Jeffrey Keller (jeffrey.keller@pbrc.edu)

NCT Registration: NCT02913664
} 
AD. The Risk Reduction for Alzheimer's Disease (rAD) trial is designed to study the independent and combined effects of intensive pharmacological control of blood pressure and cholesterol and exercise training on neurocognitive function. Six hundred and forty cognitively normal older adults age 60 to 85 years with hypertension and increased risk for dementia will be enrolled. Participants are randomized into one of four intervention group for two years: usual care, Intensive Reduction of Vascular Risk factors (IRVR) with blood pressure and cholesterol reduction, exercise training (EX), and IRVR+EX. Neurocognitive function is measured at baseline, 6, 12, 18, and 24 months; brain MRIs are obtained at baseline and 24 months. We hypothesize that both IRVR and EX will improve global cognitive function, while IRVR+EX will provide a greater benefit than either IRVR or EX alone. We also hypothesize that IRVR and EX will slow brain atrophy, improve brain structural and functional connectivity, and improve brain perfusion. Finally, we will explore the mechanisms by which study interventions impact neurocognition and brain. If $\operatorname{rr} \mathrm{AD}$ interventions are shown to be safe, practical, and successful, our study will have a significant impact on reducing the risks of $\mathrm{AD}$ in older adults.

\section{Keywords}

Alzheimer's Disease; Reduction of Vascular Risk factors; Exercise; Cognition; Brain structure

\section{Introduction}

\subsection{Alzheimer's Disease (AD) Risk and Cardiovascular Disease}

Given the rapidly aging population [1], the prevention and treatment of age-related dementias, such as $\mathrm{AD}$, presents as one of the most significant healthcare challenges of our times. Epidemiologic [2-4], laboratory, clinical [5-8], and translational studies, including genome-wide association studies [9-11], suggest that the onset and progression of agerelated dementias are multifactorial and influenced by modifiable risk factors [12-14]. With paucity of pharmacologic treatment for age-related dementias, identification of potentially modifiable risk (or protective) factors and an understanding of the effect of modifying these factors on incidence and progression of dementia is pivotal [14-16].

The presence of cardiovascular risk factors such as hypertension, hypercholesterolemia, and physical inactivity in mid-to-late life is associated with cognitive decline [17-19] and increased risk of all-cause dementia [20-22]. Older adults are particularly affected by these risk factors, with $>80 \%$ of older adults having one or more risk factors [23-25]. These cardiovascular risk factors share common pathophysiologic mechanisms of neural-vascular interactions or coupling through which cerebral atherosclerosis, cerebral small vessel disease, disruption of the blood brain barrier, and brain hypoperfusion may occur, leading to neuronal damage or dysfunction $[12,26]$. Furthermore, hypertension and hypercholesterolemia may affect brain amyloid-Beta $(\mathrm{A} \beta)$ and tau clearance through brain lipid homeostasis and blood flow regulation, further increasing AD risk [27-30].

The treatment of hypertension (HTN) and hypercholesterolemia for prevention of cognitive decline has been previously explored in clinical trials, but as secondary outcomes and with inconsistent findings [31, 32]. Aggregation of these data in recent meta-analyses and 
systematic reviews show a reduction in all-cause dementia risk by $13 \%$ with treatment of HTN [33], and 29\% with treatment of hypercholesterolemia [28]. Anti-hypertensives and statins have been shown to be safe in older adults and do not have deleterious effects on cognition [28, 32]. The benefits of intensive blood pressure (BP) and cholesterol lowering for preventing cardiovascular disease (CVD) and stroke have been demonstrated in a series of large clinical trials [34-36]. In addition to lowering risk of CVD and stroke, the recent Systolic Blood Pressure Intervention Trial (SPRINT) also showed lowering of combined clinical endpoints of incident mild cognitive impairment and dementia in the intensive BP control arm [37]. At present, the optimal goal of BP and blood lipid level reductions for dementia prevention remains uncertain. There is a strong suggestion from the current data that lowering of BP and/or treatment with statins may prevent or slow cognitive decline in older adults at risk for dementia [38].

\subsection{Lifestyle interventions for cardiovascular risk}

It is estimated that up to a half of $\mathrm{AD}$ cases may be related to physical inactivity, vascular and other potentially modifiable risk factors [39, 40]. Large population studies have indicated that the incidence of all-cause dementia may be decreasing, perhaps a reflection of multiple risk and protective factors, including higher educational levels, better control of cardiovascular risk factors and disease, and healthier lifestyles among older adults [41, 42]. An accumulating body of evidence from epidemiological, cross-sectional, and neuroimaging studies suggests that exercise is an important lifestlye strategy that is beneficial for cognitive health with aging. Prospective studies of non-demented older adults have shown a consistent relationship between higher levels of physical activity and reduced risk of dementia and cognitive decline, even after controlling for confounding factors such as education, vascular risk, and co-morbidities[43]. In cross-sectional studies, older adults who report greater engagement in physical activity earlier in life have a reduced risk of cognitive impairment and also perform better on tests of global cognitive function and executive function, compared to those who report low levels of activity[44, 45]. Neuroimaging studies have shown that older adults with higher fitness levels have reduced cerebral atrophy[46], higher grey matter density in pre-frontal and temporal regions[47], and larger hippocampal volumes[48]. These findings are encouraging and suggest that reducing modifiable risk factors may prevent or delay the onset of cognitive decline and AD [16, 49-51]. However, randomized controlled trials (RCTs) of the effects of exercise on cognitive in healthy older adults have had inconsistent findings. Although some meta-analyses reported improvements in cognitive outcomes in response to exercise training [52], a recent Cochrane Review that included twelve RCTs concluded that in healthy older adults, improving cardiorespiratory fitness does not result in improvements in cognitive function[53]. More recent RCTs of aerobic training using very similar protocols have also reported variable results[54, 55]. More exercise intervention studies, with longer follow-up periods, are needed to clarify the role of exercise as a preventive strategy for cognitive decline. Exercise alone, however, is unlikely to be fully effective in reducing cardiovascular risk factors once they have been established [35, 56]. It is also possible that exercise and/or reducing vascular risk factors each may have small to modest effects, but an integrated multimodal intervention of intensive pharmacological treatment and exercise may have additive and synergistic effects $[12,57-59]$. The efficacy and effectiveness of an integrated strategy in the prevention and 
treatment of CVD and other non-communicable chronic diseases are well established [35, 56], but not well understood for prevention of cognitive decline or age-related dementia.

\section{Methods}

\subsection{Summary/overall design}

The objective of the rrAD study is to test the hypothesis that exercise combined with intensive pharmacological reduction of vascular risk factors (IRVR) over a period of 2 years provides greater benefits for neurocognitive function than either exercise or IRVR alone. The secondary objectives include assessment of improvements in domain specific neurocognitive function, patient-reported outcome measures of mental and physical health and healthrelated quality of life, whole brain and hippocampal volume, global and regional brain perfusion, white matter lesions, brain white matter microstructural integrity, and neural network functional connectivity. Finally, the underlying mechanisms by which the study interventions impact brain structure and function will be explored by collecting blood biomarkers. rrAD is a multi-center, placebo controlled, randomized, trial with a $2 \times 2$ factorial design. The study will enroll 640 cognitively normal older adults with hypertension and risk for dementia. Participants will be randomized to receive usual care (UC), intensive reduction of vascular risk factors (IRVR) with pharmacologic treatment of BP and cholesterol, moderate-to-vigorous intensity aerobic exercise (EX), or a combination of intensive reduction of vascular risk factors and exercise (IRVR+EX). We hypothesize that both IRVR and EX alone will improve global cognitive function, while the combination, IRVR+EX will provide a greater benefit than either IRVR or EX alone.

\subsection{Recruitment}

Six hundred and forty adults, age 60-85 years are being recruited from the Baton Rouge, Dallas, Kansas City, and St. Louis areas where the prevalence rate of CVD is high relative to other regions in the U.S. [60, 61]. The rrAD study website (www.rradtrial.org), newsletters, social media, and study flyers are being used to raise the awareness of the study among the local population and healthcare networks. Each site is also using multiple and diverse means of recruitment including print media, local radio station and television, local healthcare and research registries, and partnerships with health organizations, e.g., Young Men's Christian Associations (YMCA), insurance organizations, and Alzheimer's Association chapters. Both the existing population within the clinical practice of each site, and individuals from the community are used to identify eligible participants. The rrAD study pays special attention to raising awareness of the study among local community healthcare networks through an outreach program to primary care physicians (PCPs) and other health care professionals. This strategy is intended to stimulate patient referral while minimizing potential population selection bias and healthy participant effect. The rrAD study strives to include minorities to reflect population distributions at each site.

\subsection{Inclusion/Exclusion Criteria}

The inclusion/exclusion criteria for the rrAD study are based on the principle of maximizing the trial external and internal validity by targeting target those who have elevated risk for AD. The inclusion criteria are: 1) HTN with a systolic blood pressure (SBP) between 130 
and $180 \mathrm{mmHg}$, or SBP between 110 and $130 \mathrm{mmHg}$ when being treated for HTN; 2)

Subjective memory concerns or having a first degree relative (parent or sibling) with diagnosis of dementia, without having significant cognitive impairment themselves (MiniMental State Exam [MMSE] $\geq 26$ and no clinical diagnosis of dementia) [62]; 3) Sedentary lifestyle (i.e., not meeting the CDC physical activity standard of 30 minutes of moderate activity 5 days per week or 20 minutes of vigorous activity 3 days per week) [63], but with physical ability to undergo exercise training and/or able to walk at least 10 minutes without pain; 4) Fluent in English and adequate visual and auditory acuity to allow neuropsychological testing; 5) Have a primary care physician (PCP). The exclusion criteria are: 1) Clinically documented history of stroke, focal neurological signs, or other major cerebrovascular diseases based on clinical judgment or previous brain imaging; 2) Diagnosis of AD or other dementia, or significant neurologic diseases such as Parkinson's disease, seizure disorder, multiple sclerosis, history of severe head trauma, or normal pressure hydrocephalus; 3) Evidence of severe major depression (Geriatric Depression Scale [GDS] $\geq$ 12) [64] or clinically significant psychopathology, (e.g. psychosis and schizophrenia); 4) Unstable heart disease based on clinical judgment (e.g., heart attack/cardiac arrest, cardiac bypass procedures within previous 6 months and congestive heart failure), or other severe medical conditions; 5) History of atrial fibrillation and evidence on echocardiogram with active symptoms of persistent palpitation, dizziness, history of syncope, chest pain, dyspnea, orthopnea, shortness of breath at rest, or paroxysmal nocturnal dyspnea within the past 6 months; 6) resting heart rate (HR) of < 30 or $>110 \mathrm{bpm}$; taking class I or III anti-arrhythmic drugs or clinical concerns for safely participating in exercise and lowering BP; 7) SBP $\geq 180$ $\mathrm{mmHg}$ and/or diastolic BP (DBP) $\geq$ than $110 \mathrm{mmHg}$; 8) Orthostatic hypotension, defined as the third measurement of standing SBP $<100 \mathrm{mmHg}$ after standing from a sitting position; 9) History of autoimmune disorder (i.e., systemic lupus erythematosus, rheumatoid arthritis or polymyalgia rheumatica); 10) Diabetes mellitus with hemoglobin A1C $>7.5 \%$ or requiring insulin treatment; 12) Regular smoking within the past year; 13) Women with a potential for pregnancy, currently pregnant, or lactating; 14) Enrolled in another investigational drug or device study, currently or with the past 2 months; 15) Body mass index (BMI) >45; 16) allergy to angiotensin receptor blocker or statins; 17) Abnormal screening laboratory tests; (e.g., liver function tests [ALT or AST $>3 \times \mathrm{ULN}$ ], creatine phosphokinase $>3 \times \mathrm{ULN}$, estimated glomerular filtration rate $<30 \mathrm{mg} / \mathrm{dl}$ or hematocrit $<$ $28 \%$ ); 18) Presence of a medical condition limiting estimated survival to < 3 years; 19) Any other condition(s) judged by the study investigator to be medically inappropriate, risky, or likely to cause poor study compliance.

Approval for this study was obtained from the Human Subjects Committee at Pennington Biomedical Research Center (PBRC)-Baton Rouge, The University of Texas Southwestern Medical Center and Texas Health Presbyterian Hospital Dallas- Dallas (UTSW/IEEM), University of Kansas Medical Center-Kansas City (KUMC), and Washington University School of Medicine- St. Louis (WashU).

\subsection{Methodological Considerations for Primary Outcome Measures}

The primary objective of the study is to determine the independent and combined effects of exercise and intensive vascular risk reduction (BP and LDL-C) on neurocognitive function in 
older adults who have increased risk for developing Alzheimer's disease. We have chosen the ADCS-PACC and the NIH Toolbox Cognition battery because the tests included in these batteries have been shown to be sensitive and reliable in detecting age-related cognitive decline and have been widely used in studies involving older adults. In addition, systemic reviews and meta-analyses of large observational studies and clinical trials have indicated that potential effects of physical activity and vascular risk reduction on neurocognitive function are manifested primarily in the memory and executive domains which are a focus in the ADCS-PACC and NIH Toolbox Cognition battery. Finally, using each of these assessment batteries will help ensure the data from the current study will be able to be placed in context of the study outcomes in other ongoing trials.

\subsubsection{Study assessments}

See Table 1 for a complete schedule of all study assessments.

\subsubsection{Screening}

2.5.1.1. Prescreening: The prescreening questionnaire is completed over the phone or in person at a study site upon receiving a phone call or e-mail from a potential participant. Prescreening involves explanation of study aims, gathering demographic information, collection of a brief medical and medication history, a brief physical activity questionnaire, and evaluation of eligibility [63].

2.5.1.2. Screening visits: Following prescreening, suitable individuals are invited to attend an informed consent session and two onsite screening visits. The screening assessments are divided over two visits and include informed consent, vital signs, electrocardiogram, blood collection, physical exam, screening for cognitive impairment and depression, and obtaining medical history.

\subsection{Vital signs}

2.5.1.2.1.1. Sitting heart rate and blood pressure: $\mathrm{HR}$ and $\mathrm{BP}$ are measured on the right arm in a sitting position after resting quietly for at least 5 minutes using a standard automated BP device (Welch Allyn Connex® Vital Signs Monitor series 6000). Three measurements are performed approximately 1 minute apart. An average of the second and third measurements is calculated for data analysis.

2.5.1.2.1.2 Standing heart rate and blood pressure: Participants are asked to stand up to measure HR and standing BP. Three sequential measurements are performed immediately after the participant stands. Participants are eligible if the SBP remains $\geq 100 \mathrm{mmHg}$ based on the third measurement.

2.5.1.2.3. Height, weight and BMI: Participants are asked to remove their shoes for height and weight measurements. Height is recorded in $\mathrm{cm}$ and weight in kilograms. BMI is calculated using the standard equation (kilograms $/$ meter $^{2}$ ).

\subsection{Demographics, family history, past medical history and current medications:} Demographic information including birth month and year, sex, race, ethnicity, primary 
language, years of education, employment status, profession, marital status, current living situation (i.e., independent, assisted, etc.), residence type, zip code, and right or left handedness (self-report) is obtained. Family history of parental, sibling and children diagnosis of dementia, their age at diagnosis and status (if applicable) are obtained. A detailed history of the participant's current and past medical conditions (including cardiovascular disease) is also obtained. Current medications are recorded by participant (or significant other) report, current pharmacy action profiles, and verification of medications documented in the medical record.

2.5.1.2.5. Screening for cognitive impairment and depression: The participants take the MMSE, a commonly used brief screening tool for cognitive impairment [62]. MMSE evaluates orientation, memory, attention, concentration, naming, repetition, comprehension, and ability to create a sentence and standardized figures. The total MMSE score ranges from 0 to 30 with a lower score indicative of greater cognitive impairment. Individuals with a score of $\geq 26$ are eligible for the study.

2.5.1.2.6. Geriatric Depression Scale (GDS): The GDS (16 item) is used to assess the participant's depressive symptoms. Scores under 11 are eligible for this study. [64].

2.5.1.2.7. Non-fasting clinical blood chemistry: Non-fasting blood samples $(\sim 10 \mathrm{ml})$ are collected for measurements of hemoglobin A1C, creatine phosphokinase and hematocrit. Blood collection and processing procedures and clinical laboratory tests are conducted by Quest Diagnostics (Secaucus, NJ), serving as a central clinical chemistry laboratory for the rrAD Study.

2.5.1.2.8. Physical/neurological exam and electrocardiogram: A medically qualified professional performs a complete physical and neurological exam. A 12-lead electrocardiogram is performed and interpreted by a qualified physician to assess the presence of cardiac arrhythmia/atrial fibrillation, ischemic cardiac disease, history of myocardial infarction and/or left ventricular hypertrophy.

2.5.2. Outcome Assessments-Individuals who complete screening and satisfy all inclusion/exclusion criteria are offered the opportunity to participate in the study. A detailed timeline of all study assessments is available in Table 1. All baseline assessments are completed within 6 weeks prior to randomization. Intervention assessments at 6,12 and 18 months are completed within a visit window of two-weeks. End of study assessment (24 months) are completed within 3 weeks of 24 months from randomization.

2.5.2.1. Neuropsychological testing and questionnaires: Neurocognitive function is assessed using the Alzheimer's Disease Cooperative Study Preclinical Alzheimer Cognitive Composite (ADCS-PACC) battery and the NIH-Toolbox Cognition Battery [65, 66]. The ADCS-PACC battery [65] consists of the total recall from the Free and Cued Selective Reminding test, delayed paragraph recall from the Logical Memory subtest from the Wechsler Memory Scale IV, WAIS-R Digit-Symbol Substitution, and the MMSE. The ADCS-PACC produces a composite score determined from its components using an established normalization method [67]. Each of the 4 component scores is divided by the 
baseline sample standard deviation of that component to form standardized z-scores, which are summed to form the total composite.

The NIH-Toolbox Cognition Battery [66] is an omnibus assessment that tests various cognitive domains. The following NIH Toolbox tests are used in rrAD to supplement the ADCS-PACC battery: list sorting working memory, flanker inhibitory control and attention task, dimensional change card sort test, and pattern comparison processing speed.

Patient Reported Outcomes (PRO) of physical and mental health, cognition and healthrelated quality of life are assessed using NIH PROMIS instruments [68, 69]. This assessment includes a variety of self-reported questionnaires regarding global health, physical function, sleep disturbance, sleep-related impairment, anxiety, depression, anger and applied cognitive function. T-scores for each PROMIS domain are used as outcome variables, with a mean of $50(\mathrm{SD}=10)$ in the general population. $\mathrm{PRO}$ are measured at baseline, $6,12,18$ and 24 months.

The Cognitive Change Index (20 item) is a self-rating scale of subjective cognitive decline in memory and executive over the past 5 years, including the severity of any current problems [70]. The neuropsychological tests and questionnaires are completed at baseline, 6, 12, 18 and 24 months. Every effort is made to schedule participants at the same time of day for all testing time points. Participants are instructed to eat and take their normal and study medications prior to each assessment.

All neurocognitive testing is performed by trained neuropsychometricians who are required to have at least one year of experience with cognitive testing of older adults or approval from the investigators. In addition, each psychometrician completes a detailed training and scoring procedure that includes at least two supervised test administrations by a site identified master tester. Quarterly quality control checks for each study site is performed to ensure accurate scoring, and protocols undergo periodic central review, with feedback to sites as needed.

2.5.2.2. Fasting Clinical Chemistry: Fasting blood samples $(\sim 40 \mathrm{ml})$ are collected after overnight fasting (9-12 hours) for measurements of comprehensive metabolic panel and a lipid panel. Genetic specimens (DNA and RNA) banking are obtained only from participants who consent to this procedure. Whole blood, plasma and serum $(\sim 30 \mathrm{ml})$ are aliquoted and stored at $-80^{\circ} \mathrm{C}$ for future assays, "omics" data analyses, and long-term storage. Blood collection and processing procedures and clinical laboratory tests are conducted by Quest Diagnostics. Fasting blood chemistry is collected at baseline, 12 and 24 months.

2.5.2.3 Monitoring for adverse events / Symptom Check: Clinical symptoms associated with low BP, such as dizziness, headaches or back pain, are documented at each clinical assessment and milestone visit (baseline, 6, 12, 18 and 24 months).

2.5.2.4. Exercise testing/Peak $\mathrm{VO}_{2}$ : Exercise testing of peak $\mathrm{VO}_{2}$ with the measurement of BP, 12 lead ECG, $\mathrm{HR}$, oxygen uptake is performed: 1) to measure peak $\mathrm{VO}_{2}$; and 2) to determine the safety of individual subjects while receiving exercise training. Peak $\mathrm{VO}_{2}$ 
achieved during the test is used as the index of aerobic capacity. It is not expected that all participants will be able to achieve ACSM criteria for a $\mathrm{VO}_{2 \max }$ [71]. Following the selection of treadmill pace and start grade (baseline only), the test begins at $40-60 \%$ of the participants HR reserve. Grade is increased $2 \%$ per 2 minutes until the participant elects to terminate the test, the lead tester evaluates the presence relative or absolute indications for test termination or if maximal $\mathrm{VO}_{2}$ criteria have been reached [71]. Maximal effort on the test is determined when at least three of the following criteria is achieved: 1) plateau in $\mathrm{VO}_{2}$ ( $<100 \mathrm{ml}$ change from last $60 \mathrm{sec}$. avg. of previous stage) [72, 73]; 2) resting respiratory exchange ratio $>1.1 ; 3$ ) rating of perceived exertion > 17 on 6-20 scale [74], and/or 4) heart rate $>90 \%$ of age-predicted max. Fitness is assessed at baseline, 12 and 24 months.

2.5.2.5. 10 meter walk dual task: The rrAD study uses a $10 \mathrm{~m}$ walk dual task (walking with and without distraction) to assess the subject's gait velocity and ability to perform two simultaneous tasks $[75,76]$. Non-distracted gait consists of the average of two timed $10 \mathrm{~m}$ walk times at normal walking speed. Distracted gait consists of asking participants to spell a 5-letter word backwards while walking at normal walking speed on the same $10 \mathrm{~m}$ course. Scoring includes the average walking time and whether the word was spelled correctly for both distracted gait attempts. Dual task is assessed at baseline, 12 and 24 months.

\subsubsection{Assessment of physical function: The Short Physical Performance Battery} (SPPB) is used to assess physical function and disability risk [77]. It includes three tasks that evaluate lower extremity function: 1) a standing balance task, 2) a gait speed task, and 3) a chair rise task. The score for each task ranges from 0-4, and the composite SPPB score is based on the sum of the three tasks (range 0-12). Physical function is assessed at baseline, 12 and 24 months.

\subsubsection{Assessment of Daily Physical Activity: Yale Physical Activity Survey (YPAS)}

for older adults is a self-reported questionnaire to determine the type, amount and pattern of physical activity/exercise in older adults [78]. This survey is used to monitor changes in subject's physical activity in daily life at baseline, 6, 12, 18 and 24 months.

2.5.2.8. Magnetic Resonance Imaging (MRI): MRI measurements of brain structure and function are conducted at baseline and at 24 months or before the end of participation. The rrAD study adapted the core sequences of the AD Neuroimaging Initiative but with optimization [79, 80]. MRI is performed using 3T MRI scanners (UTSW on a Philips Achieva scanner and on a GE Discovery MR 750W scanner, KUMC on a Siemens Skyra scanner, WashU on a Siemens Prisma scanner, and PBRC on a GE Discovery MR 750W scanner). Image quality at each site has been certified by an MRI physicist (DCZ) based on both human brain and phantom scans. The sequences used include: $3 \mathrm{D} \mathrm{T}_{1}$-weighted highresolution images to quantify brain morphometry; $T_{2}$ fluid-attenuated inversion recovery to measure white matter lesions; diffusion tensor imaging to assess brain white matter microstructural integrity and structural connectivity; resting-state functional MRI to assess neural network functional connectivity; arterial spin labeling to assess brain regional cerebral blood flow; $\mathrm{T}_{2}{ }^{*}$-weighted images to detect hemorrhage; and 2D phase contrast images to measure arterial blood flow into the brain. Participants are instructed to not 
consume caffeine within two hours prior to the MRI scan. Total MRI time is approximately 1 hour per session to minimize participant burden. If MRI data are not usable, the participant can be asked to return for a repeat scan.

2.5.3. Staff training and standardization of data collection-Face-to-face training sessions were conducted at each study site during site initiation for quality assurance and quality control purposes. The rrAD study Clinical Coordinating Center (UTSW), the Data Coordinating Center (UTSW), the Biomarker Core (UTSW, and the Imaging Coordinating Center (Michigan State University) provide guidelines and technical support to all study sites as needed. Each study site organizes training and refresher training sessions, including remediation in specific areas targeted by quality control monitoring.

Study personnel performing outcome assessments are blinded to group assignment. The rrAD study chief statistician performing primary data analysis is also blinded. Intervention staff, site safety officers, and individuals not involved in outcome measures are considered unblinded to address safety concerns or adverse events.

2.5.4 Data collection-Data are collected and stored in the Research Electronic Data Capture (REDCap), a web-based, electronic data capture tool hosted on a secure, password protected, HIPAA-compliant server at UTSW medical center [81]. Case-report-forms (CRFs) are entered into the REDCap database using web entry. In addition to sightverification, the site study coordinators, the Clinical Coordinating Center (CC), and the Data Coordinating Center (DCC) use REDCap's data quality control module, which reports missing values, validation errors, and outliers as well as reports developed by the $\mathrm{CC}$ and DCC. The Biomarker Core receives blood, plasma, and serum samples from each site with unique participant IDs. Biospecimens are shipped in batches to ensure safe receipt, and samples are banked upon arrival according the NIA Biospecimen Best Practice Guidelines for the Alzheimer's Disease Centers (https://www.alz.washington.edu/ BiospecimenTaskForce.html). Biospecimens are electronically recorded and all timed samples from one participant will be analyzed concurrently to ensure within-participant data integrity. Samples are, however, aliquoted in the event assay replicates are needed. All MRI data with unique participant IDs are transferred via a secure web-based Globus research data management service software (https://www.globus.org/) in DICOM format to the Imaging Coordinating Center (ICC) located at the Michigan State University (MSU) within 24 hours after the participant is scanned. After receiving the scan information and data from a site, the ICC team will complete the data quality control (QC) within three business days. The QC ensures (1) that the image data are uploaded to the server appropriately; (2) MRI data match participant's ID, date and protocol parameters; (3) the completeness of the scan; (4) all images are transferred with HIPAA-compliant, study-specific identifiers and do not contain identifiable participant-specific information; (5) image quality (imaging distortion, artifacts and signal-to-noise ratio) for all scans; and (6) run preliminary analyses, such as the temporal signal-to-noise ratio and motion parameters for fMRI, to determine if the images have sufficient quality for data analysis. The site will be notified if any severe image artifacts are found and recommendations will be provided if a participant should be rescanned and/or if phantom QC scans should be performed. 


\subsection{Randomization}

Participants are randomized into one of four study arms stratified by study site, blood pressure, age and sex using a constant blocking factor (UC, EXEX, IRVR, and IRVR+EX). Randomization assignments are managed centrally using REDCap after all the baseline procedures are completed and the participant qualifies for the study.

\subsection{Interventions}

2.7.1. Usual Care (UC)-Participants in the UC follow their PCP's recommendations for BP and cholesterol control. Participants in this group may or may not be treated with one or more pharmacological agents at the initial visit. If SBP is not $<150 \mathrm{mmHg}$ at any time point, participant will be asked to visit with their PCP for a BP medication titration.

Instructing participants not to exercise is unethical. Therefore, to encourage study retention the investigators will provide all UC and IRVR participants with an optional home-based stretching and balance program, as well as access to a stretching and balance program at their local YMCA. In the event participants perform the home-based exercises, attend the YMCA classes or begin another form of exercise on his or her own, we will assess these changes in physical activity using the Yale Physical Activity Survey at baseline, 6, 12, 18 and 24 months (see Table 1). The home-based stretching program consists of a standardized set of nine exercises focused primarily on joint range of motion and flexibility that has been implemented in previous RCTs of exercise training [82]. They are instructed to perform the stretching and balance exercises 3 times per week and to gradually increase the frequency over a period of 3-4 months to 4-5 times per week. Participants are instructed to record the number of times and duration per week they perform the stretching and balance exercises on a calendar provided by the study staff, which is reviewed monthly by study staff. To enhance study retention, participants are also encouraged to attend monthly stretching exercise classes hosted by each study site over the 24 months, which are led by study staff or exercise trainers. No additional education or support is provided to the UC group beyond the homebased stretching program and the monthly classes.

2.7.2. Aerobic Exercise (EX)—Participants in EX only arm follow their PCP's recommendations for BP and cholesterol management as in the UC group. In addition, they perform a structured, moderate-to-vigorous aerobic exercise training and supervised by exercise professionals at local exercise facilities. Each study site is at liberty to engage with community facilities or use their own exercise facility to conduct the training. Identified facilities have appropriate safety protocols and exercise trainers with nationally-recognized certification (e.g., ACSM, ACE) or appropriate education (exercise physiology degree, clinical licensure, etc.). Each study site follows the direction of their IRB as to what and how facilities are approved for use. Figure 1 shows the template for rrAD EX training by week during the intervention period. An exercise training log, safety tips, and instructions for using a HR monitor is provided to participants at the first orientation visit. The first orientation visit also includes the first in-person training session to instruct participants in proper exercise intensity for their physical fitness levels. Treadmill walking or jogging, cycling, stepping (including recumbent), and similar aerobic modalities are acceptable. 
During Weeks 1-4, all participants receiving the EX intervention are supervised by a trainer for every session ( 3 times per week). Trainers fit participants with a HR monitor and help participants to exercise in the appropriate target HR zone and at the associated Borg Rating of Perceived Exertion (RPE; 6-20) [74]. Trainers help participants understand what training HR zones are associated with what perceived exertion levels during weeks $1-4$, so that when the participants begin to exercise independently, they can appropriately adjust their work rate based on perceived exertion rather than target HR zone. Trainers ask participants their RPE every 5-10 minutes while monitoring HR and teach the participants to adjust intensity accordingly by speed, grade, resistance, etc. as appropriate. Trainers instruct participants how to appropriately warm-up and cool-down at least 5 minutes at every session.

Participants are instructed to engage in structured aerobic physical activity 3 or more times per week to complete the minimal requirement of prescribed weekly exercise duration.

Per Figure 1, intensity gradually increases over the first 3 months of the study to allow participants to gradually and safely adapt to exercise. Exercise logs given to each participant have information on target effort and target HR zone for each month of exercise. The exercise program is prescribed individually, based on the maximal HR obtained during peak $\mathrm{VO}_{2}$ testing at baseline. At the beginning of the study, participants exercise at a light-tomoderate intensity, an RPE of 10-12 or 55-65\% of HRmax. By Week 5 participants begin to exercise at a moderate intensity, an RPE of 12-14 or 65-75\% of HRmax. By Week 9, participants are encouraged to gradually incorporate more vigorous levels of exercise as able with support and supervision of their trainer, including RPE of $14-17$ or $65-85 \%$ of HRmax.

Exercise duration or volume is gradually increased over the first 6 months. By Month 6, all participants receiving the EX intervention are expected to achieve the goal of 160 minutes of moderate-to-vigorous aerobic exercise per week at the prescribed intensity, including $\sim 5$ minutes warm-up and cool-down periods at each exercise session. This goal was selected to encourage participants to meet or exceed public health recommendations [71]. Participants unable to exercise continuously will perform intermittent bouts (minimum 10 min each) until the target is reached. During Weeks 5-12, participants are supervised at least 1 session per week. Beginning at Week 13, participants are allowed to exercise at home or at another training location, if the trainer and/or certified study staff confirm that participants are able to judge their exercise intensity with RPE. Participants are instructed to work with their trainer at least 1 session per month for the duration of the 24-month intervention period. The participant is expected to record the duration of exercise (not including warm up and cool down) and their overall judgment of RPE in an exercise log at every exercise session. Study staff regularly check these logs at least once per month for protocol compliance. Adherence is measured as the percentage of total minutes per week of prescribed exercise.

2.7.3. Intensive Reduction of Vascular Risk Factors (IRVR)-Participants in the IRVR group follow the rAD BP and cholesterol lowering algorithms for treatment of hypertension and hypercholesterolemia (Figure 2). Individuals randomized to this group also receive the home-based stretching and balance exercise program as in UC group to account for potential "placebo" effects of aerobic exercise training as described in section 2.7.1. At the first orientation/vascular clinic visit, a 30-90-day supply of study medication (the amount of drug dispensing will be site specific) and a brochure about the study medication, 
safety tips, and instructions for taking study medications is provided to the participants. Potential drug-drug interactions and adjustment or replacement of current BP or cholesterol lowering medications (if they are taking any) is discussed with the participants and any questions are addressed before the initiation of drug therapy. As for the UC group, instructions for performing stretching and balance training properly, safety tips, and a stretching and balance training log, are provided at the first orientation visit.

The rrAD study uses an evidence-based protocol to lower SBP (goal $<130 \mathrm{mmHg}$ ) and cholesterol (goal LDL-C < $70 \mathrm{mg} / \mathrm{dL}$ )(Figure 2)[35, 83, 84]. Calcium channel blocker (CCB) amlodipine and angiotensin II receptor blocker (ARB) losartan are the first line antihypertensives for IRVR group because of their effectiveness in reducing BP and reduction in CVD risk and safety in large clinical trials [84-86]. Low dose thiazide diuretic chlorthalidone, potassium-sparing diuretic spironolactone, or alpha 1-adrenergic receptor inhibitor doxazosin will be added if needed to reach the BP goal. For participants already being treated for hypertension with SBP of 110 to 130, their antihypertensives will be replaced with amlodipine and losartan to maintain or achieve a SBP $<130 \mathrm{mmHg}$ if applicable based on a clinical assessment.

For treating hypercholesterolemia, atorvastatin $80 \mathrm{mg}$ daily is used for the IRVR participants with a fasting LDL-C $\geq 70 \mathrm{mg} / \mathrm{dL}$. For individuals who have LDL-C $<70 \mathrm{mg} / \mathrm{dL}$, and are not on any statins, atorvastatin is not initiated. Those with LDL-C $<70 \mathrm{mg} / \mathrm{dL}$ on a statin, their statin is switched to atorvastatin with an equivalent dose as the current statin if applicable based on a clinical assessment. Figure 2 describes the detailed algorithm for cholesterol management. The study clinician may adjust the dose, stop or change antihypertensives to reach SBP goal of $<130 \mathrm{mmHg}$ in the interest of participant safety. Once the SBP goal is achieved, the anti-hypertensive regimen may be intensified further if DBP remains $\geq 100 \mathrm{mmHg}$ at a single visit or $\geq 90 \mathrm{~mm} \mathrm{Hg}$ at two successive visits.

Medication adjustments are made on an individual basis if a participant develops side effects. For example, if a participant develops myalgias or elevation in CPK, the atorvastatin dose may be reduced or stopped depending on the severity of the side effects. Study medication may be restarted after the associated side effects are reduced or adequately addressed. Once randomized, if a participant is no longer able to continue with the study, he/she may be followed for intent-to-treat purposes.

2.7.4. IRVR+EX-Participants in the IRVR+EX group follow the BP and cholesterol lowering algorithm as well as the structured aerobic exercise training program, as described above.

2.7.5. Intervention compliance and adherence monitoring-Participants are encouraged to stay with the intervention/s they are randomized to. However, potential changes in lifestyle or other health-related behaviors may occur (e.g., participants in the UC or the IRVR group may start aerobic exercise). Any changes in the health-related behavior or medication, which may impact study outcome, are reviewed, discussed and documented during regular study visits. 
Treatment effects of BP lowering are monitored at each study visit. Participants are asked to return study medications and bottles, used and unused, at each visit. Pill counts are performed to calculate percentage of pills taken/dispensed to document drug adherence in the IRVR and IRVR+EX groups. Exercise adherence is recorded as the percentage of the total number of minutes exercised (recorded in $\log$ ) per week relative to the number prescribed in the EX and IRVR+EX groups. The rrAD study goal is $>80 \%$ treatment adherence for both IRVR and EX interventions. Potential effects of intervention adherence on study outcome measures will be assessed as secondary data analysis.

Monthly phone or in-person follow-up and three-month clinic visits to monitor study safety and compliance are conducted with all participants to maintain a same level of study contact across the study groups. At each visit, participants are asked about any changes in their health or medications to monitor potential influences on study outcome measures.

\subsection{Strategies for participant retention}

Every effort is made to provide a friendly clinic environment for study visits, foster personal relationships between study staff and participants, and minimize study burden. Written instructions for follow-up visits are provided to participants and reminder calls are made before each study visit to increase attendance. Exercise training programs and locations are flexible to fit each participant's needs and preferences. Alterations in exercise mode, duration, or intensity are made as necessary to maintain the overall training pattern and "dose" among participants and across study sites. Time constraints and medical conditions can hinder exercise patterns in older adults. If participation falls below target (i.e., 80\%), site staff or the exercise trainer works with the participant to identify barriers and increase adherence. Strategies are employed to help the participants overcome these barriers to adherence (i.e., planning a schedule, changing exercise modes if injured, etc.). Exercise training may be restarted after an interruption due to medical or other personal issues. These and other strategies such as goal setting, positive reinforcement, self-monitoring, regular follow-up, and motivational interviewing, are used to enhance study adherence. Retention strategies are also employed if a participant is not compliant with their IRVR study visits or medication.

Similar to interventions to increase adherence with exercise, study staff work with participants to develop personalized strategies to encourage visit attendance and medication adherence. Each of the 4 sites has experience in successfully conducting exercise and pharmocological studies in the elderly and will utilize different approaches that have proven to work well in the past at each site. The overarching theme to these approaches is to provide a "customer service" approach that continually focuses on reducing participant burden as much as possible, addressing participant questions and concerns in a rapid manner, and maintaining as much uniformity and consistency as possible in terms of interactions with the participant. Examples include trying to maintain continuity of coordinator-participant interactions, providing sufficient lead time and reminders for scheduled visits, and promptly responding the emails and/or phone calls from the participant. In addition, study sites will be hosting thank you events for study participants and the rrAD study provides a modest 
stipend every 6 months for all participants, following the completion of each outcome assessment visit (See Table 1.).

\subsection{Sample size estimation and data analysis plan}

2.9.1. Sample size estimation-The rrAD study is designed to detect changes in neurocognitive function among the intervention groups and to test the central hypothesis that benefits of IRVR+EX on improving global neurocognitive function is greater than those of UC, IRVR alone, or EX alone. The composite $\mathrm{z}$ score of global cognition will be obtained by conversion of individual test scores to the standardized $\mathrm{z}$ scores, then averaged to obtain a composite score. Confirmatory factor analysis (CFA) will be used to validate the proposed domain-specific and global cognition scores. Sample size and power estimates were conducted using a linear mixed models analysis of variance (ANOVA) design estimating effect size using Cohen's $d$ for the interaction of EX by IRVR combinations. Several covariance structures (AR (1)), compound symmetry, unstructured, etc.) for the repeated measures were considered with time points of 5, 3 and 2. Table 2 presents the results of sample size and power estimates based on the covariance structure of compound symmetry. For primary outcome of global neurocognitive function (5-time points over 2 years), with a correlation of 0.5 between repeated measures and an alpha level of 0.05 , group sample sizes of 128 in each of EX by IRVR combinations (accounts for a potential 20\% dropout rate) will achieve $82.4 \%$ power to detect a standardized difference of $\mathrm{d}=0.28$ in changes in global neurocognitive function between the IRVR $+\mathrm{EX}$ and the UC group $(2 \times 2$ factorial design $)$.

\subsubsection{Analysis plan}

2.9.2.1. General considerations: Baseline and demographic characteristics will be summarized overall and by treatment group. Summaries will be provided for continuous and categorical measures. All measures will be screened for outliers and transformation may be performed to normalize the data, if necessary. Residual and multicollinearity diagnostics will be performed to assess model assumption. Unless otherwise noted, all tests of treatment effects and interactions will be conducted at a 2-sided alpha level of 0.05; 2-sided confidence intervals will be displayed with a $95 \%$ confidence level. The primary analysis consists of a linear mixed model with the interaction terms of treatments and time as the primary predictor variables. We assume that changes in global cognitive function over 2-yrs will be linear and this assumption will be confirmed with plots of individual growth curves. All analyses will follow the modified intent-to-treat (mITT) principle unless otherwise specified [87]. An ITT analysis is an analysis of data by groups to which the subjects are assigned by random allocation, even if the subject does not take the assigned treatment, does not receive the correct treatment, or otherwise does not follow the protocol. A mITT analysis is an ITT analysis for all participants who have a baseline and at least 1 post baseline measure. Secondary analyses will consist of adjustments for potential effects of covariates on outcome measures such as baseline cognitive score, APOE4 genotype, peak $\mathrm{VO}_{2}$, and treatment effects of BP and lipids to reveal a potential "dose-response" relationship.

2.9.2.2. Safety Mornitoring and DSMB: Potential side effects of antihypertensive and atorvastatin will be reviewed and discussed with participants during the enrollment. Participants will be asked to inform the study clinician or staff immediately if side effects 
occur and persist. Participants will be monitored and interviewed during each of the followup visits to ensure that any symptoms are addressed early and properly. The rrAD data safety and monitoring board (DSMB) will review and approve the study protocol including the plan for safety monitoring and reporting. DSMB will also monitor the study data integrity and any adverse effects which may occur during the study, and evaluate the progress of the trial, including assessments of data quality, timeline of recruitment and accrual, retention, participant risk versus benefit, performance of the trial sites, and other factors that can influence study progression and outcome. The DSMB will receive safety report quarterly and meet every six months with rrAD investigators to monitor study safety and progress, advise the NIH about the study performance, and make recommendations regarding study continuation and protocol changes.

2.9.2.3. Interim analysis: The medications and supervised aerobic exercise training used in the rrAD study are widely used clinical interventions to improve cardiovascular health in older adults. However, the benefits of these interventions on neurocognitive function have not been demonstrated conclusively, and the potential effect size is likely to be small to moderate based on observational studies and meta-analysis of a few of small RCTs. In addition, the benefits of these interventions on brain structure and function, if they manifest, are likely cumulative over time. Considering the potential moderate effects of the rrAD study on neurocognitive function, the study team has decided not to conduct an interim efficacy analysis. However, the rrAD study DSMB may require an interim safety data analysis based on overall safety profile, work-in-progress report, adverse event or serious adverse event monitoring.

2.9.2.4. Missing data: The MRI involves two assessments, one at baseline and one at 24 months, thus limiting the range of analytic strategies. We plan to use a maximum likelihood based general linear models for analyzing imaging outcomes [88]. Intracranial volume will be included as a covariate. The validity of the Missing at Random assumption will be examined and baseline measurements will be included as covariates for modeling and data analysis. If loss to follow-up is related to the unobserved cognitive outcome then our results may be biased. Again, modeling assumptions and sensitivity analysis may be considered if necessary. mITT and ITT analysis will be used to handle all behavior and other missing data.

\section{Discussion}

The rrAD study is vital to providing clinicians, public health organizations, and policy makers information on independent and combined importance of IRVR and EX treatments. rrAD is the first study utilizing a $2 \times 2$ factorial design to determine both the independent and combined effects of EX and IRVR on neurocognitive function and AD biomarkers in older adults who are at high risk for $\mathrm{AD}[38,89]$. Using an integrated systems approach, this project aims to reduce several age-related co-morbidities (hypertension, hypercholesterolemia, and physical inactivity) simultaneously to achieve maximal gain to improve brain health in older adults. In addition, the selected design is potentially deployable as it can utilize community physicians and health resources to deliver the IRVR and EX treatments. 
The rrAD study will also be the first study using state-of-the-art neuroimaging and blood biomarkers along with detailed neurocognitive measures to determine the independent and combined effects of EX and IRVR on brain pathophysiological changes related to AD. Extensive studies have previously reported that hypertension, elevated cholesterol and/or physical inactivity are associated with brain atrophy, white matter hyperintensities and brain amyloid burden in older adults [90-93]. rrAD will provide direct evidence whether the proposed interventions alter the trajectory of brain pathophysiological changes, and whether IRVR+EX has greater impacts than IRVR or EX alone on brain structure and function, thus indicating a potential cause-effect relationship between the presence of vascular risk factors and brain pathophysiology.

Finally, evidence from rrAD will have immediate implications for target BP levels and intensity of cholesterol management to prevent cognitive decline in older adults. Our approach for vascular risk reduction will serve as the standard (e.g., the medications to be used, treatment effect, and safety evaluations) for future $\mathrm{AD}$ prevention trials. If $\operatorname{rrAD}$ interventions are proven to be practical and successful, our study may lead to revision of the guidelines to intensify cardiovascular risk factor control for prevention of $\mathrm{AD}$ in older adults.

\section{Funding:}

This study was supported by the National Institutes of Health grant R01-AG49749 and KL2TR002367

\section{Abbreviations:}

$\begin{array}{ll}\text { A } \beta & \text { Amyloid-Beta } \\ \text { AD } & \text { Alzheimer's Disease } \\ \text { ADCS-PACC } & \begin{array}{l}\text { Alzheimer's Disease Cooperative Study Preclinical Alzheimer } \\ \text { Cognitive Composite }\end{array} \\ \text { BP } & \text { Blood Pressure } \\ \text { CVD } & \text { Cardiovascular disease } \\ \text { FLAIR } & \text { fluid-attenuated inversion recovery } \\ \text { EX } & \text { Aerobic exercise } \\ \text { HR } & \text { Heart rate } \\ \text { HTN } & \text { hypertension } \\ \text { KUMC } & \text { University of Kansas Medical Center } \\ \text { LDL-C } & \text { Low-density lipoprotein cholesterol } \\ \text { IRVR } & \text { = Intensive Pharmacological Reduction in Vascular Risk } \\ \text { mITT } & \text { modified intent-to-treat }\end{array}$

Contemp Clin Trials. Author manuscript; available in PMC 2019 April 01. 


$\begin{array}{ll}\text { MRI } & \text { Magnetic Resonance Imaging } \\ \text { NIRS } & \text { near infrared spectroscopy } \\ \text { PBRC } & \text { Pennington Biomedical Research Center } \\ \text { PCP } & \text { Primary Care Physician } \\ \text { PRO } & \text { Patient Reported Outcomes of physical and mental health } \\ \text { RCT } & \text { Randomized controlled trial } \\ \text { rrAD } & \text { Risk Reduction for Alzeimer's Disease } \\ \text { UC } & \text { Usual Care } \\ \text { UTSW } & \text { University of Texas Southwestern Medical Center } \\ \text { WashU } & \text { Washington University Medical Center } \\ \text { YMCA } & \text { Young Men's Christian Associations }\end{array}$

\section{References}

1. 2018 Alzheimer's disease facts and figures. Alzheimer's \& Dementia, 2018 14(3): p. 367-429.

2. Gelber RP, et al., Antihypertensive medication use and risk of cognitive impairment: the HonoluluAsia Aging Study. Neurology, 2013 81(10): p. 888-95. [PubMed: 23911753]

3. Seshadri S, Delaying dementia: can antihypertensives prevent Alzheimer dementia? Neurology, 2013 81(10): p. 860-2. [PubMed: 23911755]

4. Ahlskog JE, et al., Physical Exercise as a Preventive or Disease-Modifying Treatment of Dementia and Brain Aging. Mayo Clinic Proceedings, 2011 86(9): p. 876-884. [PubMed: 21878600]

5. Iadecola C, Neurovascular regulation in the normal brain and in Alzheimer's disease. Nature Reviews Neuroscience, 2004 5(5): p. 347. [PubMed: 15100718]

6. Sagare AP, Bell RD, and Zlokovic BV, Neurovascular dysfunction and faulty amyloid beta-peptide clearance in Alzheimer disease. Cold Spring Harb Perspect Med, 2012 2(10).

7. Nelson AR, et al., Neurovascular dysfunction and neurodegeneration in dementia and Alzheimer's disease. Biochim Biophys Acta, 2016 1862(5): p. 887-900. [PubMed: 26705676]

8. Ngandu T, et al., A 2 year multidomain intervention of diet, exercise, cognitive training, and vascular risk monitoring versus control to prevent cognitive decline in at-risk elderly people (FINGER): a randomised controlled trial. The Lancet, 2015 385(9984): p. 2255-2263.

9. Huang Y and Mucke L, Alzheimer mechanisms and therapeutic strategies. Cell, 2012 148(6): p. 1204-22. [PubMed: 22424230]

10. Pedersen NL, Reaching the limits of genome-wide significance in Alzheimer disease: back to the environment. Jama, 2010 303(18): p. 1864-5. [PubMed: 20460629]

11. Cuyvers E and Sleegers K, Genetic variations underlying Alzheimer's disease: evidence from genome-wide association studies and beyond. The Lancet Neurology, 2016 15(8): p. 857-868. [PubMed: 27302364]

12. Kling MA, et al., Vascular disease and dementias: paradigm shifts to drive research in new directions. Alzheimers Dement, 2013 9(1): p. 76-92. [PubMed: 23183137]

13. Morris JK, et al., Is Alzheimer's disease a systemic disease? Biochim Biophys Acta, 2014 1842(9): p. 1340-9. [PubMed: 24747741]

14. Norton S, et al., Potential for primary prevention of Alzheimer's disease: an analysis of populationbased data. The Lancet Neurology, 2014 13(8): p. 788-794. [PubMed: 25030513] 
15. Bauer UE, et al., Prevention of chronic disease in the 21 st century: elimination of the leading preventable causes of premature death and disability in the USA. The Lancet, 2014 384(9937): p. $45-52$.

16. Kivipelto $\mathrm{M}$ and Mangialasche F, Alzheimer disease: To what extent can Alzheimer disease be prevented? Nat Rev Neurol, 2014 10(10): p. 552-3. [PubMed: 25245154]

17. Dregan A, Stewart R, and Gulliford MC, Cardiovascular risk factors and cognitive decline in adults aged 50 and over: a population-based cohort study. Age Ageing, 2013 42(3): p. 338-45. [PubMed: 23179255]

18. Abete P, et al., Cognitive impairment and cardiovascular diseases in the elderly. A heart-brain continuum hypothesis. Ageing Res Rev, 2014 18: p. 41-52. [PubMed: 25107566]

19. Wysocki M, et al., Hypertension is Associated With Cognitive Decline in Elderly People at High Risk for Dementia. The American Journal of Geriatric Psychiatry, 2012 20(2): p. 179-187. [PubMed: 21814158]

20. Ligthart SA, et al., Treatment of cardiovascular risk factors to prevent cognitive decline and dementia: a systematic review. Vasc Health Risk Manag, 2010 6: p. 775-85. [PubMed: 20859546]

21. Richard E, Moll van Charante EP, and van Gool WA, Vascular risk factors as treatment target to prevent cognitive decline. J Alzheimers Dis, 2012 32(3): p. 733-40. [PubMed: 22886011]

22. Purnell C, et al., Cardiovascular risk factors and incident Alzheimer disease: a systematic review of the literature. Alzheimer Dis Assoc Disord, 2009 23(1): p. 1-10. [PubMed: 18703981]

23. Federal Interagency Forum on Aging-Related Statistics, Older Americans 2016: Key Indicators of Well-Being. 2016: Washington, DC: U.S. Government Printing Office.

24. Toth PP, Potter D, and Ming EE, Prevalence of lipid abnormalities in the United States: the National Health and Nutrition Examination Survey 2003-2006. J Clin Lipidol, 2012 6(4): p. 32530. [PubMed: 22836069]

25. Aronow WS, et al., ACCF/AHA 2011 expert consensus document on hypertension in the elderly: a report of the American College of Cardiology Foundation Task Force on Clinical Expert Consensus Documents. Circulation, 2011 123(21): p. 2434-506. [PubMed: 21518977]

26. Fotuhi M, Hachinski V, and Whitehouse PJ, Changing perspectives regarding late-life dementia. Nat Rev Neurol, 2009 5(12): p. 649-58. [PubMed: 19918254]

27. Sánchez-Ferro Á, et al., A review of the potential therapeutic role of statins in the treatment of Alzheimer's disease: current research and opinion. Neuropsychiatric Disease and Treatment, 2013 9: p. 55-63. [PubMed: 23319866]

28. Swiger KJ, et al., Statins and cognition: a systematic review and meta-analysis of short- and longterm cognitive effects. Mayo Clin Proc, 2013 88(11): p. 1213-21. [PubMed: 24095248]

29. Chu CS, et al., Use of statins and the risk of dementia and mild cognitive impairment: A systematic review and meta-analysis. Sci Rep, 2018 8(1): p. 5804. [PubMed: 29643479]

30. Schultz BG, Patten DK, and Berlau DJ, The role of statins in both cognitive impairment and protection against dementia: a tale of two mechanisms. Transl Neurodegener, 2018 7: p. 5. [PubMed: 29507718]

31. McGuinness B, et al., Statins for the prevention of dementia. Cochrane Database Syst Rev, 2016(1): p. Cd003160. [PubMed: 26727124]

32. McGuinness B, et al., Blood pressure lowering in patients without prior cerebrovascular disease for prevention of cognitive impairment and dementia. Cochrane Database Syst Rev, 2009(4): p. Cd004034. [PubMed: 19821318]

33. Peters R, et al., Incident dementia and blood pressure lowering in the Hypertension in the Very Elderly Trial cognitive function assessment (HYVET-COG): a double-blind, placebo controlled trial. Lancet Neurol, 2008 7(8): p. 683-9. [PubMed: 18614402]

34. James PA, et al., 2014 evidence-based guideline for the management of high blood pressure in adults: Report from the panel members appointed to the eighth joint national committee (jnc 8). JAMA, 2014 311(5): p. 507-520. [PubMed: 24352797]

35. Stone NJ, et al., 2013 ACC/AHA guideline on the treatment of blood cholesterol to reduce atherosclerotic cardiovascular risk in adults: a report of the American College of Cardiology/ American Heart Association Task Force on Practice Guidelines. J Am Coll Cardiol, 2014 63(25 Pt B): p. 2889-934. [PubMed: 24239923] 
36. A Randomized Trial of Intensive versus Standard Blood-Pressure Control. New England Journal of Medicine, 2015 373(22): p. 2103-2116. [PubMed: 26551272]

37. Kjeldsen SE, et al., Intensive blood pressure lowering prevents mild cognitive impairment and possible dementia and slows development of white matter lesions in brain: the SPRINT Memory and Cognition IN Decreased Hypertension (SPRINT MIND) study. Blood Pressure, 2018 27(5): p. 247-248. [PubMed: 30175661]

38. Daviglus ML, et al., NIH state-of-the-science conference statement: Preventing Alzheimer's disease and cognitive decline. NIH Consens State Sci Statements, 2010 27(4): p. 1-30.

39. Barnes DE and Yaffe K, The projected effect of risk factor reduction on Alzheimer's disease prevalence. Lancet Neurol, 2011 10(9): p. 819-28. [PubMed: 21775213]

40. Norton S, et al., Potential for primary prevention of Alzheimer's disease: an analysis of populationbased data. Lancet Neurol, 2014 13(8): p. 788-94. [PubMed: 25030513]

41. Larson EB, Yaffe K, and Langa KM, New insights into the dementia epidemic. N Engl J Med, 2013 369(24): p. 2275-7. [PubMed: 24283198]

42. Whalley LJ and Smyth KA, Human culture and the future dementia epidemic: crisis or crossroads? Neurology, 2013 80(20): p. 1824-5. [PubMed: 23596078]

43. Hamer M and Chida Y, Physical activity and risk of neurodegenerative disease: a systematic review of prospective evidence. Psychol Med, 2009 39(1): p. 3-11. [PubMed: 18570697]

44. Middleton LE, et al., Physical activity over the life course and its association with cognitive performance and impairment in old age. J Am Geriatr Soc, 2010 58(7): p. 1322-6. [PubMed: 20609030]

45. Guiney $\mathrm{H}$ and Machado L, Benefits of regular aerobic exercise for executive functioning in healthy populations. Psychon Bull Rev, 2013 20(1): p. 73-86. [PubMed: 23229442]

46. Colcombe $\mathrm{S}$, et al., Aerobic fitness reduces brain tissue loss in aging humans., in J Gerontol Biol Sci Med Sci. 2003 p. 176-180.

47. Gordon BA, et al., Neuroanatomical correlates of aging, cardiopulmonary fitness level, and education. Psychophysiology, 2008 45(5): p. 825-38. [PubMed: 18627534]

48. Erickson KI, et al., Aerobic fitness is associated with hippocampal volume in elderly humans. Hippocampus, 2009 19(10): p. 1030-9. [PubMed: 19123237]

49. Lincoln P, et al., The Blackfriars Consensus on brain health and dementia. Lancet, 2014 383(9931): p. $1805-6$.

50. Smith AD and Yaffe K, Dementia (including Alzheimer's disease) can be prevented: statement supported by international experts. J Alzheimers Dis, 2014 38(4): p. 699-703. [PubMed: 24326609]

51. Solomon A, et al., Advances in the prevention of Alzheimer's disease and dementia. J Intern Med, 2014 275(3): p. 229-50. [PubMed: 24605807]

52. Colcombe $\mathrm{S}$ and Kramer A, Fitness effects on the cognitive function of older adults: A metaanalytic study. Psychol Sci, 2003 14: p. 125-130. [PubMed: 12661673]

53. Young J, et al., Aerobic exercise to improve cognitive function in older people without known cognitive impairment. Cochrane Database Syst Rev, 2015(4): p. Cd005381. [PubMed: 25900537]

54. Vidoni ED, et al., Dose-Response of Aerobic Exercise on Cognition: A Community-Based, Pilot Randomized Controlled Trial. PLoS One, 2015 10(7): p. e0131647. [PubMed: 26158265]

55. McDaniel M, et al., Effects of cognitive training with and without aerobic exercise on cognitively demanding everyday activities. Psychol Aging, 2014 29(3): p. 717-730. [PubMed: 25244489]

56. Eckel RH, et al., 2013 AHA/ACC guideline on lifestyle management to reduce cardiovascular risk: a report of the American College of Cardiology/American Heart Association Task Force on Practice Guidelines. Circulation, 2014 129(25 Suppl 2): p. S76-99. [PubMed: 24222015]

57. Frisardi V, et al., Metabolic-cognitive syndrome: a cross-talk between metabolic syndrome and Alzheimer's disease. Ageing Res Rev, 2010 9(4): p. 399-417. [PubMed: 20444434]

58. Yaffe K, Metabolic syndrome and cognitive disorders: is the sum greater than its parts? Alzheimer Dis Assoc Disord, 2007 21(2): p. 167-71. [PubMed: 17545744]

59. Joyner MJ and Green DJ, Exercise protects the cardiovascular system: effects beyond traditional risk factors. J Physiol, 2009 587(Pt 23): p. 5551-8. [PubMed: 19736305] 
60. Fryar CD, Chen TC, and Li X, Prevalence of uncontrolled risk factors for cardiovascular disease: United States, 1999-2010. NCHS Data Brief, 2012(103): p. 1-8.

61. Mozaffarian D, et al., Heart disease and stroke statistics--2015 update: a report from the American Heart Association. Circulation, 2015 131(4): p. e29-322. [PubMed: 25520374]

62. Cockrell JR and Folstein MF, Mini-mental state examination. Principles and practice of geriatric psychiatry, 2002: p. 140-141.

63. Topolski TD, et al., The Rapid Assessment of Physical Activity (RAPA) Among Older Adults. Preventing Chronic Disease, 2006 3(4): p. A118. [PubMed: 16978493]

64. Yesavage JA, et al., Development and validation of a geriatric depression screening scale: a preliminary report. Journal of psychiatric research, 1982 17(1): p. 37-49. [PubMed: 7183759]

65. Donohue MC, et al., The preclinical Alzheimer cognitive composite: measuring amyloid-related decline. JAMA Neurol, 2014 71(8): p. 961-70. [PubMed: 24886908]

66. Weintraub S, et al., Cognition assessment using the NIH Toolbox. Neurology, 2013 80(11 Suppl 3): p. S54-64. [PubMed: 23479546]

67. Cutter GR, et al., Development of a multiple sclerosis functional composite as a clinical trial outcome measure. Brain, 1999122 (Pt 5): p. 871-82. [PubMed: 10355672]

68. Barile JP, et al., Monitoring population health for Healthy People 2020: evaluation of the NIH PROMIS® Global Health, CDC Healthy Days, and satisfaction with life instruments. Quality of Life Research, 2013 22(6): p. 1201-1211. [PubMed: 23404737]

69. Gershon RC, et al., The Use of PROMIS and Assessment Center to Deliver Patient-Reported Outcome Measures in Clinical Research. Journal of applied measurement, 2010 11(3): p. 304-314. [PubMed: 20847477]

70. Rattanabannakit C, et al., The Cognitive Change Index as a Measure of Self and Informant Perception of Cognitive Decline: Relation to Neuropsychological Tests. J Alzheimers Dis, 2016 51(4): p. 1145-55. [PubMed: 26923008]

71. American College of Sports, M., et al., ACSM's guidelines for exercise testing and prescription. 2018.

72. Proctor DN and Joyner MJ, Skeletal muscle mass and the reduction of VO2max in trained older subjects. J Appl Physiol (1985), 1997 82(5): p. 1411-5. [PubMed: 9134886]

73. Howley ET F. BD, Health Fitness Instructor's Handbook. 2nd ed, ed. Franks BD. 1995, Champaign, IL: Human Kinetics.

74. Borg G, Borg's perceived exertion and pain scales. 1998: Human kinetics.

75. Pizzamiglio S, et al., Neural Correlates of Single- and Dual-Task Walking in the Real World. Frontiers in Human Neuroscience, 2017 11: p. 460. [PubMed: 28959199]

76. Rosso AL, et al., DUAL-TASK WALKING AS A PREDICTOR FOR LATER COGNITIVE IMPAIRMENT. Alzheimer's \& Dementia: The Journal of the Alzheimer's Association, 2017 13(7): p. P1417-P1418.

77. Guralnik JM, et al., A Short Physical Performance Battery Assessing Lower Extremity Function: Association With Self-Reported Disability and Prediction of Mortality and Nursing Home Admission. Journal of Gerontology, 1994 49(2): p. M85-M94. [PubMed: 8126356]

78. Pereira MA, et al., A collection of Physical Activity Questionnaires for health-related research. Med Sci Sports Exerc, 1997 29(6 Suppl): p. S1-205. [PubMed: 9243481]

79. Weiner MW, et al., Impact of the Alzheimer's Disease Neuroimaging Initiative, 2004 to 2014. Alzheimers Dement, 2015 11(7): p. 865-84. [PubMed: 26194320]

80. Weiner MW, et al., The Alzheimer's Disease Neuroimaging Initiative 3: Continued innovation for clinical trial improvement. Alzheimers Dement, 2017 13(5): p. 561-571. [PubMed: 27931796]

81. Harris PA, et al., Research electronic data capture (REDCap)--a metadata-driven methodology and workflow process for providing translational research informatics support. J Biomed Inform, 2009 42(2): p. 377-81. [PubMed: 18929686]

82. McDaniel MA, et al., Effects of cognitive training with and without aerobic exercise on cognitively demanding everyday activities. Psychol Aging, 2014 29(3): p. 717-30. [PubMed: 25244489]

83. Allen JK, et al., Community Outreach and Cardiovascular Health (COACH) Trial: a randomized, controlled trial of nurse practitioner/community health worker cardiovascular disease risk 
reduction in urban community health centers. Circ Cardiovasc Qual Outcomes, 2011 4(6): p. 595602. [PubMed: 21953407]

84. Go AS, et al., An effective approach to high blood pressure control: a science advisory from the American Heart Association, the American College of Cardiology, and the Centers for Disease Control and Prevention. J Am Coll Cardiol, 2014 63(12): p. 1230-1238. [PubMed: 24246165]

85. Forette F, et al., The prevention of dementia with antihypertensive treatment: new evidence from the Systolic Hypertension in Europe (Syst-Eur) study. Arch Intern Med, 2002 162(18): p. 204652. [PubMed: 12374512]

86. Li NC, et al., Use of angiotensin receptor blockers and risk of dementia in a predominantly male population: prospective cohort analysis. Bmj, 2010 340: p. b5465. [PubMed: 20068258]

87. Montedori A, et al., Modified versus standard intention-to-treat reporting: Are there differences in methodological quality, sponsorship, and findings in randomized trials? A cross-sectional study. Trials, 2011 12: p. 58-58. [PubMed: 21356072]

88. Friston KJ, et al., Statistical parametric maps in functional imaging: A general linear approach. Human Brain Mapping, 1994 2(4): p. 189-210.

89. Services, U.S.D.o.H.a.H., National Plan to Addres Alzheimer's Disease: 2017 Update. 2017: Washington, DC.

90. Beauchet O, et al., Blood pressure levels and brain volume reduction: a systematic review and meta-analysis. J Hypertens, 2013 31(8): p. 1502-16. [PubMed: 23811995]

91. Hughes TM, et al., Markers of cholesterol transport are associated with amyloid deposition in the brain. Neurobiol Aging, 2014 35(4): p. 802-7. [PubMed: 24199960]

92. Rodrigue KM, et al., Risk factors for beta-amyloid deposition in healthy aging: vascular and genetic effects. JAMA Neurol, 2013 70(5): p. 600-6. [PubMed: 23553344]

93. DeCarli C, Cerebrovascular disease: Assessing the brain as an end-organ of vascular disease. Nat Rev Cardiol, 2012 9(8): p. 435-6. [PubMed: 22733217] 


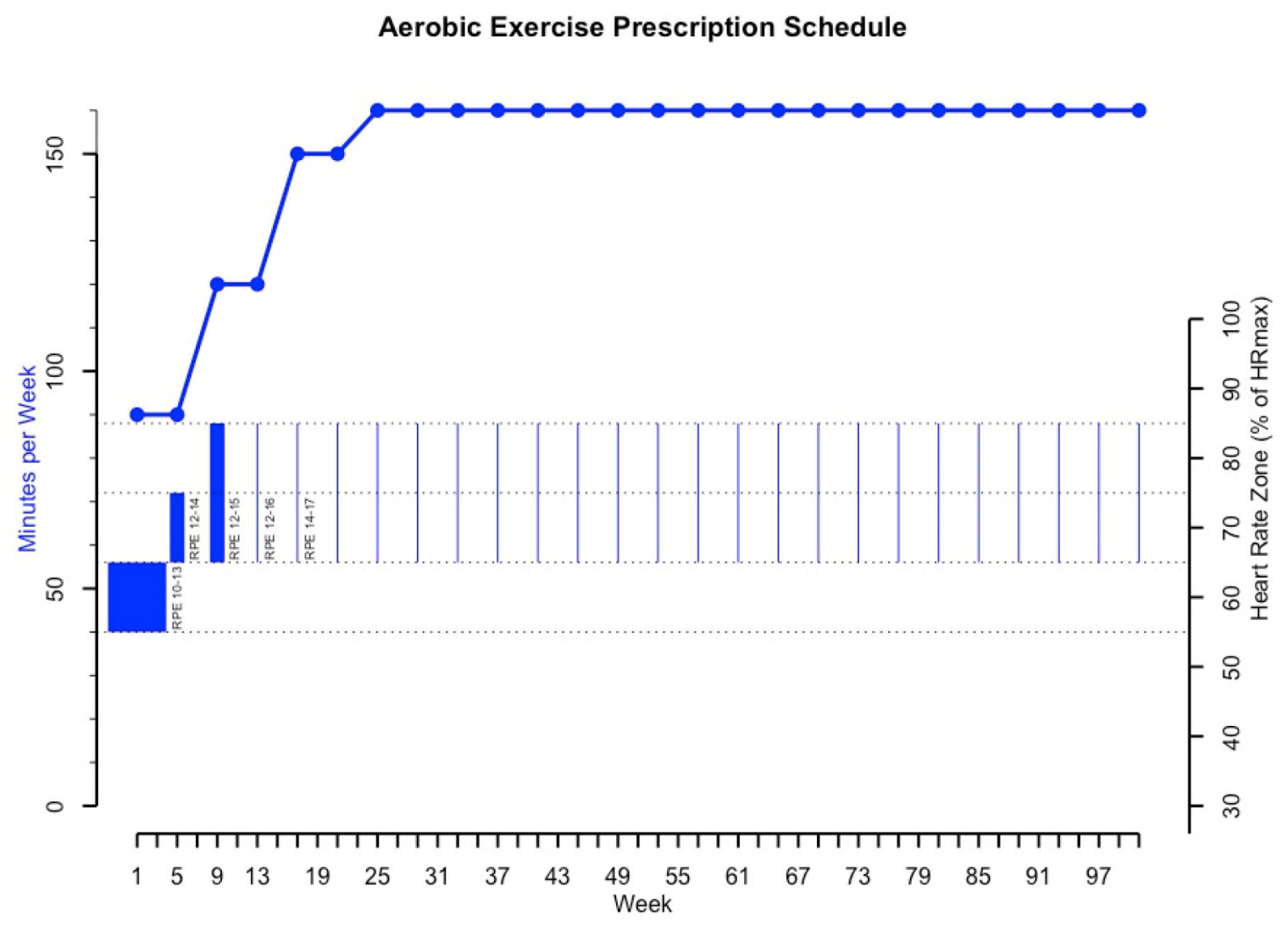

Figure 1.

rrAD Study Aerobic Exercise Training Schedule 
Start Atorvastatin $80 \mathrm{mg}$ daily if indicated. For HTN (SBP $>130 \mathrm{mmHg}$ ), Check if patients are on treatment. If no meds, initiates with amlodipine $2.5 \mathrm{mg}$ and losartan $12.5 \mathrm{mg}$ once daily.

If patients are on 1 drug, stop current meds and start amlodipine $5 \mathrm{mg}$ and losartan $\mathbf{2 5} \mathrm{mg}$ once daily. If the patients are on $\geq 2$ drug, stop current meds and start amlodipine $10 \mathrm{mg}$ and losartan $\mathbf{5 0} \mathrm{mg}$ once daily.

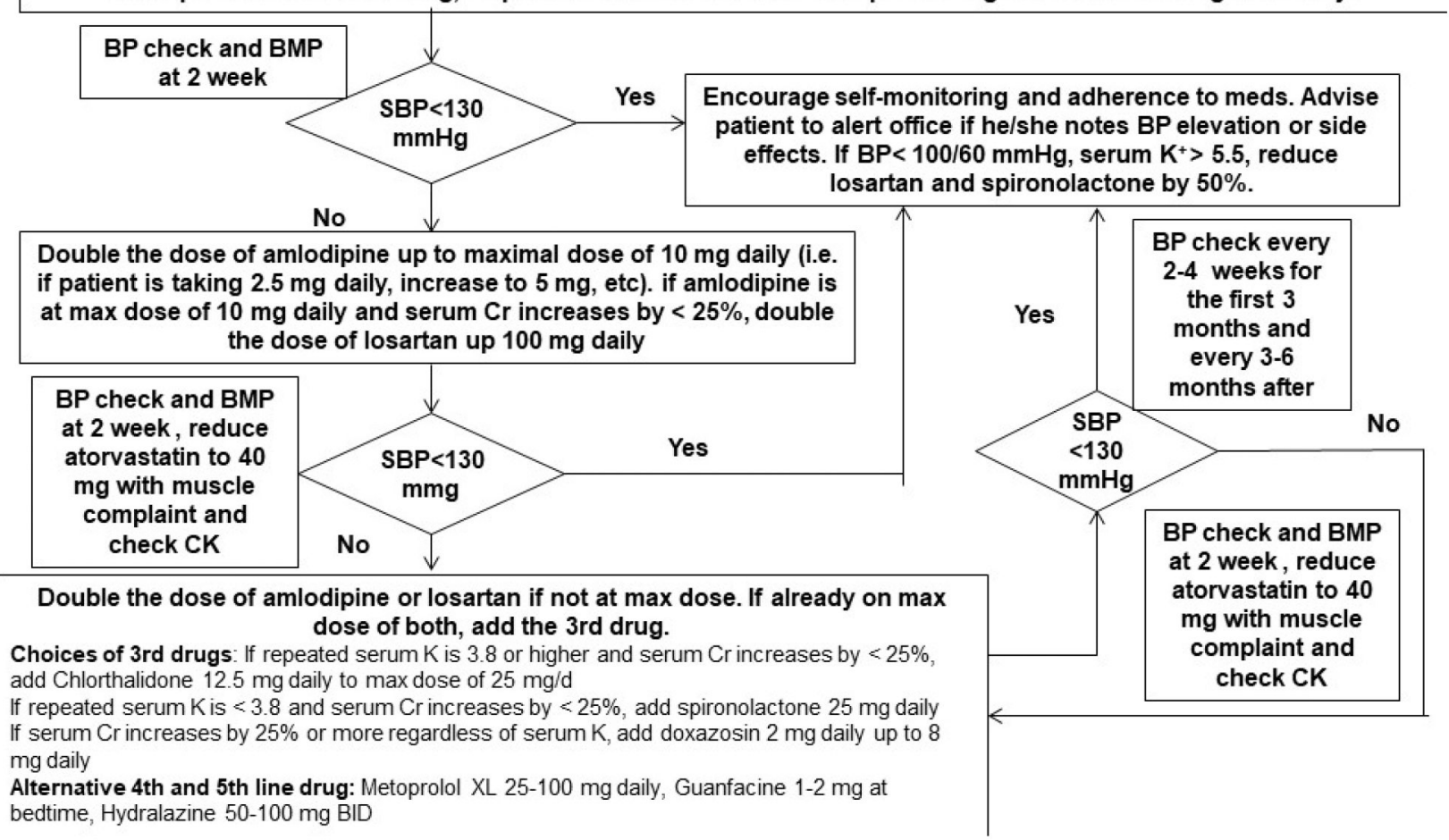

Figure 2.

Cholesterol Management in IRVR and IRVR + EX 
Table 1.

Study Assessments

\begin{tabular}{|c|c|c|c|c|c|c|c|}
\hline & Prescreening & In Person Screening & Baseline & 6 Months & 12 Months & 18 Months & $\begin{array}{c}24 \\
\text { Months/ } \\
\text { End of } \\
\text { Study }\end{array}$ \\
\hline Prescreening questionnaire & $\mathrm{X}$ & & & & & & \\
\hline Informed Consent & & $\mathrm{X}$ & & & & & \\
\hline BP Measurement & & $\mathrm{X}$ & & & & & \\
\hline MMSE & & $\mathrm{X}$ & & $\mathrm{x}$ & $\mathrm{X}$ & $\mathrm{X}$ & \\
\hline Demographics & & $\mathrm{X}$ & & & & & \\
\hline Family History & & $\mathrm{X}$ & & & & & \\
\hline Health History & & $\mathrm{X}$ & & & & & \\
\hline Medications & & $\mathrm{X}$ & & & & & \\
\hline GDS & & $\mathrm{X}$ & & & & & \\
\hline $\begin{array}{l}\text { Non-Fasting Clinical Blood } \\
\text { Chemistry }\end{array}$ & & $\mathrm{X}$ & & & & & \\
\hline Physical Exam & & $\mathrm{X}$ & & & & & \\
\hline ECG & & $\mathrm{X}$ & & & & & \\
\hline FAQ & & & $\mathrm{X}$ & & $\mathrm{X}$ & & $\mathrm{X}$ \\
\hline Neurocognitive Assessments & & & $\mathrm{X}$ & $\mathrm{X}$ & $\mathrm{X}$ & $\mathrm{X}$ & $\mathrm{X}$ \\
\hline Fasting Blood Chemistry & & & $\mathrm{X}$ & & $\mathrm{X}$ & & $\mathrm{X}$ \\
\hline Symptoms Check & & & $\mathrm{X}$ & $\mathrm{x}$ & $\mathrm{X}$ & $\mathrm{X}$ & $\mathrm{X}$ \\
\hline Exercise Test & & & $\mathrm{X}$ & & $\mathrm{X}$ & & $\mathrm{X}$ \\
\hline SPPB/Dual Task & & & $\mathrm{X}$ & & $\mathrm{X}$ & & $\mathrm{X}$ \\
\hline Yale Physical Activity Survey & & & $\mathrm{X}$ & $\mathrm{X}$ & $\mathrm{X}$ & $\mathrm{X}$ & $\mathrm{X}$ \\
\hline MRI & & & $\mathrm{X}$ & & & & $\mathrm{X}$ \\
\hline TCD & & & $X$ & & $X$ & & $X$ \\
\hline
\end{tabular}


Table 2.

\begin{tabular}{|c|c|c|c|c|}
\hline \multirow{7}{*}{ 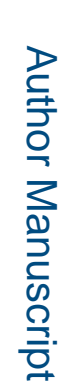 } & \multicolumn{4}{|c|}{ Sample size and power estimations ${ }^{*}$} \\
\hline & Time points & Cohen's d & Correlation & Power \\
\hline & 5 & 0.16 & 0 & $>81.6 \%$ \\
\hline & 5 & 0.24 & $0,0.3$ & $>82.4 \%$ \\
\hline & 5 & 0.28 & $0,0.3,0.5$ & $>82.4 \%$ \\
\hline & 5 & 0.31 & $0,0.3,0.5,0.7$ & $>81.1 \%$ \\
\hline & 3 & 0.21 & 0 & $>82.8 \%$ \\
\hline & 3 & 0.26 & $0,0.3$ & $>81.2 \%$ \\
\hline & 3 & 0.29 & $0,0.3,0.5$ & $>81.0 \%$ \\
\hline & 3 & 0.32 & $0,0.3,0.5,0.7$ & $>81.6 \%$ \\
\hline & 2 & 0.25 & 0 & $>80.7 \%$ \\
\hline & 2 & 0.29 & $0,0.3$ & $>82.0 \%$ \\
\hline 己 & 2 & 0.31 & $0,0.3,0.5$ & $>81.6 \%$ \\
\hline $\overrightarrow{\hat{0}}$ & 2 & 0.33 & $0,0.3,0.5,0.7$ & $>81.6 \%$ \\
\hline 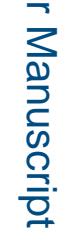 & \multicolumn{4}{|c|}{ Alpha $=0.05 ; \mathrm{N}=128 /$ group; compound symmetry. } \\
\hline
\end{tabular}

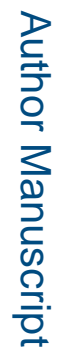

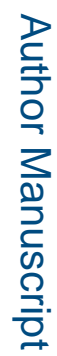

